\title{
Heat capacity in doped graphene under magnetic fields: the role of spin splitting
}

\author{
F Escudero, J S Ardenghi, P Jasen
}

IFISUR, Departamento de Física (UNS-CONICET), Av. Alem 1253, B8000CPB

Bahía Blanca, Argentina

Instituto de Física del Sur (IFISUR, UNS-CONICET)

Av. Alem 1253, B8000CPB Bahía Blanca, Argentina

E-mail: federico.escudero@uns.edu.ar

\begin{abstract}
We study the electronic heat capacity in doped graphene under magnetic fields. The partition function is calculated considering only the thermal excitations in the last occupied energy levels. Due to the large energy separation between the Landau levels (LLs) and the Zeeman splitting, at low temperatures the heat capacity is dominated by the spin excitations in the last occupied LL. Correspondingly the heat capacity oscillates with maximum amplitude at half filling of each LL. At higher temperatures the inter-LLs excitations dominate the heat capacity, with maximum amplitude at full filling factors. The oscillation amplitudes are compared with the phonon heat capacity $C_{p}$. It is shown that the spin induced heat capacity oscillations have a maximum amplitude approaching $3 \%$ of $C_{p}$, whereas for the interLLs excitations the maximum amplitude is only $0.1 \%$ of $C_{p}$. These amplitudes decrease in the presence of impurities, although the effect is appreciable if the LLs broadening is bigger than the excitation energies.
\end{abstract}




\section{Introduction}

Due to its unique characteristics, in the past decade graphene has become one of the most promising two dimensional material (2D) [1, 2]. Consisting in a bidimensional hexagonal lattice made of carbon atoms, graphene is remarkable for its strength [3, 4, 5] and high electrical conductivity [6, 7]. The electronic properties of graphene are unique among 2D systems: the valence and conduction bands touch at the Fermi energy [8], where the density of states is zero, making graphene a semiconductor with zero band gap [9]. At energies close to the Fermi level, the electrons behave as massless Dirac fermions [10, 11] with a Fermi velocity of about $10^{6} \mathrm{~m} / \mathrm{s}$ [12]. The thermal properties in graphene, although similar to graphite, also show unique features [13, 14, 15, 16]. For instance, phonons dominate the specific heat, even at very low temperatures [17].

Under magnetic fields, the thermal properties are expected to depend on the Landau levels (LLs) of the system. In graphene, at low energies the LL scale as $\sqrt{n}$ and thus are not equidistant [18]. Moreover, their cyclotron energy is remarkably high, compared to the conventional 2D electron gas [19]. Hence magnetic related effects can be seen in graphene even at relatively high temperatures [20, 21, 22]. Moreover, at low occupancy the spin splitting of the energy levels is much smaller than the LLs difference, so thermal spin excitations are expected to dominate at low temperatures. This feature can have a noticeable effect in the electronic heat capacity, which is expected to oscillate as a function of the magnetic field [23], much like the oscillations in the magnetization [24] or the conductivity [25]. The study of these oscillations has proved to be a powerful mapping tool, as their amplitudes and frequencies depend on the material properties [26]. Some aspects of these oscillations in the specific heat have been recently studied. In [27], the electronic specific heat is analysed in undoped and slighty-doped graphene, where the specific heat is shown to depend on the spin excitations between holes and electrons. In [28], the specific heat is studied at relatively high temperatures, in which case the spin splitting is safely ignored. There is not, however, an analysis of the electronic heat capacity in doped graphene at low temperatures, where the partial occupancy and the spin splitting of the LLs play an important role.

Traditionally, the heat capacity is obtained by working in the grand canonical ensemble, in which case at fixed electron density one must obtain the chemical potential oscillations with the magnetic field. These oscillations are usually small in three dimensional metals, and can be neglected in most cases [23. However, in two dimensional materials like graphene the chemical potential oscillations can be significant, especially at low temperatures [29, 30]. In general, the chemical potential oscillations can only be obtained numerically [31, limiting the analytical analysis. In this work we present an alternative way by working in the canonical ensemble, considering only the excitations of the last occupied energy levels in the ground state. We show that this approach leads to a simple analytical expression for the heat capacity, where the partial occupation of the last energy level is naturally taken into account. Furthermore, a clear distinction can be made for the contributions of the spin and LLs excitations to the heat 
capacity.

We have organized this work as follow: in Sec. 2 we describe the model used to obtain the partition function at low temperatures, taking into account only the thermal excitations of the last occupied energy levels. In Sec. 3 we obtain the electronic heat capacity, separating the contributions due the spin and LLs excitations. We discuss in detail the heat capacity oscillations, as well as the role of the spin and LLs excitations at low and high temperatures. We also compare the magnitude of the oscillations with the phonon contribution to the heat capacity, and discuss the damping of the oscillations due to impurities effects. Finally, our conclusions follow in Sec. 4.

\section{The Model}

We consider graphene in the long wavelength approximation, where electrons behave as massless relativistic fermions with an effective Hamiltonian [9]

$$
H=v_{\mathrm{F}}\left(\xi \sigma^{x} p_{x}+\sigma^{y} p_{y}\right)
$$

where $v_{\mathrm{F}} \sim 10^{6} \mathrm{~m} / \mathrm{s}$ is the Fermi velocity, $\boldsymbol{\sigma}=\left(\sigma^{x}, \sigma^{y}\right)$ are the Pauli matrices acting in the sublattices $A$ and $B$ of graphene, and $\xi= \pm 1$ for the $K$ and $K^{\prime}$ valleys. Under a perpendicular magnetic field $B$, the energy spectrum can be found using the Peierls substitution $\boldsymbol{p} \rightarrow \boldsymbol{p}-e \boldsymbol{A}$, where $\boldsymbol{A}$ is the vector potential. Then, considering the Zeeman

effect, the Landau levels (LLs) are [32] $\varepsilon_{n, s}^{\lambda}=\lambda v_{\mathrm{F}} \sqrt{2 e \hbar B n}-s \mu_{\mathrm{B}} B$, where $\lambda= \pm 1$ for the conduction and valence band, and $s= \pm 1$ for the spin up and down (we shall assume a g-factor $g \simeq 2$, which is a good approximation in graphene [33]). The LLs do not depend on the $\xi$ index and are identical for both $K$ and $K^{\prime}$ valleys. Thus each LL has a degeneracy $D=2 A n_{B}$, where $A$ is the graphene area and $n_{B}=B e / h$ is the flux density [18. Special care is needed for the $n=0 \mathrm{LL}$, which is shared by holes and electrons. The degeneracy determines the filling factor $\nu=n_{e} / n_{B}$, where $n_{e}$ is the electron density.

Assuming doped graphene with an electron density $n_{e}$, in the ground state the electrons fill the lowest conduction energy levels. For simplicity we will consider $n_{e}$ and $n_{B}$ such that $\nu>2$ and thus the $n=0 \mathrm{LL}$ is always filled. The last occupied level is, in general, partially filled with $D \Theta$ electrons, where we define $\Theta=\nu / 2$ - floor $[\nu / 2]$ as the occupancy factor $(0 \leq \Theta \leq 1)$. At low temperatures, the only non-negligible thermal excitations are between the last occupied energy levels. Hence it is convenient to write the system energy $E$ as an excitation to the ground state energy $U$, such that $E=U+\Delta E$, where $\Delta E$ is the excitation energy. Then the partition function reads

$$
Z=e^{-\beta U} \sum_{\Delta E} C_{\Delta E} e^{-\beta \Delta E}
$$

where $\beta=1 / k_{\mathrm{B}} T$ and $C_{\Delta E}$ is the combination factor that accounts for the number of ways the excitation $\Delta E$ can occur, considering that the electrons are indistinguishable. The summation in $Z$ must be restricted to the condition of constant electron density and that each LL can be occupied at most with $D$ electrons. 

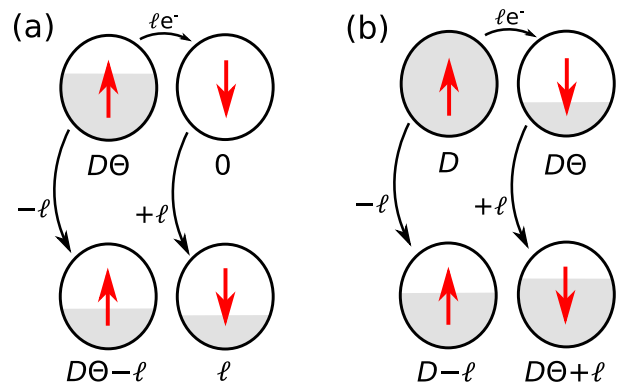

Figure 1. Schematic representation of the possible spin excitations in the last occupied energy levels. Each LL has degeneracy $D$ and their occupancy is determined by the factor $0 \leq \Theta \leq 1$. The two cases shown correspond to: (a) excitations from the last partially occupied LL with spin up to the same empty LL with spin down, and (b) excitations from the last fully occupied LL with spin up to the same partially occupied LL with spin down. In both cases, in the top it is represented the ground state occupation and in the bottom the occupation after the excitation of $\ell$ electrons.

We can distinguish two types of excitations, with different energy scales. One type is the excitations between the last occupied LL with different spin, where $\Delta E=2 \mu_{\mathrm{B}} B$. The other type is the excitations between different LLs, with $\Delta E \sim \Delta \varepsilon_{\mathrm{L}}$, where $\Delta \varepsilon_{\mathrm{L}}$ is the energy difference between the last LLs occupied in the ground state. Considering the valley degeneracy and that each LL can be occupied with both spins, we can say $\Delta \varepsilon_{\mathrm{L}} \sim v_{\mathrm{F}} \sqrt{2 e \hbar B}(\sqrt{\nu+2}-\sqrt{\nu-2}) / 2$. Thus for usual electron densities $n_{e} \sim 10^{12}$ $\mathrm{cm}^{-2}$ and strong magnetic fields $B>1 \mathrm{~T}$, we always have $\Delta \varepsilon_{\mathrm{L}} \gg 2 \mu_{\mathrm{B}} B$. In the following we obtain the partition function for both types of excitations.

\subsection{Spin excitations}

At very low temperatures, such that $e^{-\beta 2 \mu_{\mathrm{B}} B} \ll 1$, there are only spin excitations. There could be two situations for the spin excitations between the last occupied LL, as shown schematically in Fig. 1, corresponding to the two cases when the last occupied LL has spin up or down. We shall define $q=$ floor $[\nu / 2]$, so the last occupied LL has spin up when $q$ is odd and spin down when $q$ is even. For $\ell$ excited electrons, we need to calculate the combination factor $C_{\ell}$. Given that the particles are indistinguishable, each LL with occupancy $c$ can be occupied in $\left(\begin{array}{l}D \\ c\end{array}\right)$ ways, where $\left(\begin{array}{l}n \\ k\end{array}\right)=n ! / k !(n-k)$ ! is the binomial coefficient. Thus for $\ell$ excited electrons we have

$$
C_{\ell}=\left(\begin{array}{c}
D \\
D-\ell
\end{array}\right)\left(\begin{array}{c}
D \\
D_{q}+\ell
\end{array}\right)
$$

where we defined $D_{q}=D\left[1+(-1)^{q}(2 \Theta-1)\right] / 2$, taking into account both cases $q$ even and $q$ odd. Following Fig. 1, $D \Theta$ electrons can be excited when $q$ is odd, whereas $D-D \Theta$ electrons can be excited when $q$ is even. Hence the partition function is

$$
Z_{\mathrm{S}}=e^{-\beta U} \sum_{\ell=0}^{D_{q+1}} C_{\ell} e^{-2 \ell \beta \mu_{\mathrm{B}} B}=e^{-\beta U} z_{\Theta} z_{\mathrm{S}},
$$


where $z_{\Theta}=\left(\begin{array}{c}D \\ D \Theta\end{array}\right)$ and

$$
z_{\mathrm{S}}={ }_{2} F_{1}\left(-D,-D_{q+1}, D_{q}+1, e^{-2 \beta \mu_{\mathrm{B}} B}\right),
$$

where ${ }_{2} F_{1}$ is the hypergeometric function. Notice that the term $z_{\Theta}$ comes from the number of ways the last LL can be partially occupied, whereas the term $z_{\mathrm{S}}$ comes from the excitations between the same LL with different spin.

\subsection{Landau level excitations}

We now consider the excitations between different LLs, so $\Delta E \sim \Delta \varepsilon_{\mathrm{L}}$, where $\Delta \varepsilon_{\mathrm{L}}$ is the LL difference between the last occupied states. Considering the spin splitting, to calculate the partition function one would need to consider the excitations between 6 energy levels, corresponding to the three different LLs whose excitation give $\Delta E \sim \Delta \varepsilon_{\mathrm{L}}$, each with different spin. Thus one would need to consider not only two states excitations, but also multiple states (3 or more states) in which the energy difference is still of order $\Delta \varepsilon_{\mathrm{L}}$, resulting in a lengthy and complicated calculation for the partition function. However, we can simplify the problem by considering that when the thermal excitations between different LLs become appreciable, the spin splitting of the LLs can be neglected, given that $\Delta \varepsilon_{\mathrm{L}} \gg 2 \mu_{\mathrm{B}} B$. In this way we can study the excitations between LLs by ignoring the Zeeman effect. Then, instead of the excitations between 6 states, we only need to consider excitations between 3 different LLs.

Without spin splitting, the conduction band LLs are $\varepsilon_{n}=v_{\mathrm{F}} \sqrt{2 e \hbar B n}$ and each LL has degeneracy $2 D$, with the $n=0$ LL being shared by holes and electrons. Thus there is a change of LL at filling factors $\nu=2(2 n+1)$. It is convenient to define $\nu_{G}=\nu-2$ as the filling factor of the LLs $n \geq 1$. Then the last fully occupied LL in the ground state is $n=q_{0}$, where $q_{0}=$ floor $\left[\nu_{G} / 4\right]$. Considering $\nu>2$, the partial occupancy of the last $\mathrm{LL}$, in the ground state, is $\Theta_{0}=\nu_{G} / 4-$ floor $\left[\nu_{G} / 4\right]$. The possible excitations between the last occupied LLs are schematically shown in Fig. 2. In the first case, Fig. 2(a), electrons from the last fully occupied LL $\varepsilon_{q_{0}}$ are excited to the next partially occupied

LL $\varepsilon_{q_{0}+1}$. In the second case, Fig. 2(b), electrons from the last partially occupied LL $\varepsilon_{q_{0}+1}$ are excited to the next empty LL $\varepsilon_{q_{0}+2}$. For $\ell$ excited electrons, the corresponding combination factors are

$$
\begin{aligned}
C_{1, \ell} & =\left(\begin{array}{c}
2 D \\
2 D-\ell
\end{array}\right)\left(\begin{array}{c}
2 D \\
2 D \Theta_{0}+\ell
\end{array}\right) \\
C_{2, \ell} & =\left(\begin{array}{c}
2 D \\
2 D \Theta_{0}-\ell
\end{array}\right)\left(\begin{array}{c}
2 D \\
\ell
\end{array}\right) .
\end{aligned}
$$

Defining $\Delta \varepsilon_{1}=\varepsilon_{q_{0}+1}-\varepsilon_{q_{0}}$ and $\Delta \varepsilon_{2}=\varepsilon_{q_{0}+2}-\varepsilon_{q_{0}+1}$, then $D_{1}=2 D-2 D \Theta_{0}$ electrons can be excited with $\Delta \varepsilon_{1}$, whereas $D_{2}=2 D \Theta_{0}$ electrons can be excited with $\Delta \varepsilon_{2}$. Thus the partition function is

$$
Z_{\mathrm{L}} e^{\beta U}=\sum_{\ell=1}^{D_{1}} C_{1, \ell} e^{-\beta \ell \Delta \varepsilon_{1}}+\sum_{\ell=1}^{D_{2}} C_{2, \ell} e^{-\beta \ell \Delta \varepsilon_{2}} .
$$



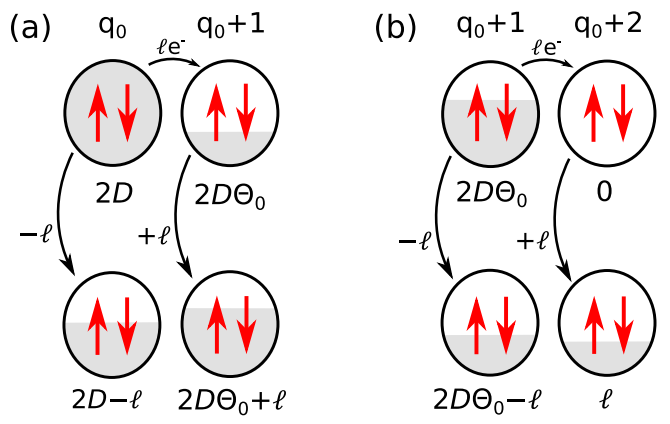

Figure 2. Schematic representation of the possible LLs excitations in the last occupied energy levels. Without spin splitting, each LL has degeneracy $2 D$, considering them occupied with both spins as shown. In the ground state, the last LL fully occupied is $n=q_{0}$, and the partial occupancy of the next LL $n=q_{0}+1$ is determined by $0 \leq \Theta_{0} \leq 1$. The two cases shown correspond to (a) excitations $\varepsilon_{q_{0}} \rightarrow \varepsilon_{q_{0}+1}$ and (b) excitations $\varepsilon_{q_{0}+1} \rightarrow \varepsilon_{q_{0}+2}$. In the top it is represented the ground state occupation and in the bottom the occupation after the excitation of $\ell$ electrons.

We can write $Z_{\mathrm{L}}=e^{-\beta U} z_{\Theta_{0}}\left(z_{\mathrm{L}}-1\right)$, where $z_{\Theta_{0}}=\left(\begin{array}{c}2 D \\ 2 D \Theta_{0}\end{array}\right)$ and

$$
\begin{aligned}
z_{\mathrm{L}}= & { }_{2} F_{1}\left(-2 D,-D_{1}, D_{2}+1, e^{-\beta \Delta \varepsilon_{1}}\right) \\
& +{ }_{2} F_{1}\left(-2 D,-D_{2}, D_{1}+1, e^{-\beta \Delta \varepsilon_{2}}\right)-1 .
\end{aligned}
$$

Like in the spin excitations case, the term $z_{\Theta_{0}}$ is related to the number of ways the last LL can be partially occupied, ignoring the spin splitting. The term $z_{\mathrm{L}}$ is due to the excitations between the last occupied LLs. Notice that $z_{\mathrm{L}}$ depends on two hypergeometric functions because of the two possible LL excitations in the last occupied states.

\subsection{Total partition function}

The total partition function, considering the spin and LLs excitations, is $Z=Z_{\mathrm{S}}+Z_{\mathrm{L}}$.

We can factorize $Z$ by noticing that when $Z_{\mathrm{L}}$ becomes relevant we have $z_{\Theta} z_{\mathrm{S}} \rightarrow z_{\Theta_{0}}$ (see Appendix), and thus $Z=e^{-\beta U} z_{\Theta} z_{\mathrm{S}} z_{\mathrm{L}}$. In this way we separate the different contributions to the partition function, which will prove useful in the analysis of the heat capacity. It is worth mentioning that the model used to obtain $Z$, considering only the thermal excitations of the last occupied energy levels, can be extended to other two dimensional systems besides graphene. The only difference would be the expression for the Landau levels. For instance, in buckled honeycomb structures such as silicene, the valley degeneracy is broken in the presence of a perpendicular electric field [34, 35]. As a result the LLs are split in each valley. Hence, as done with the spin excitations, one could analyse the inter-valley excitations and separate its contribution to the partition function. This could be useful to understand the valley dependent properties in $2 \mathrm{D}$ materials. 

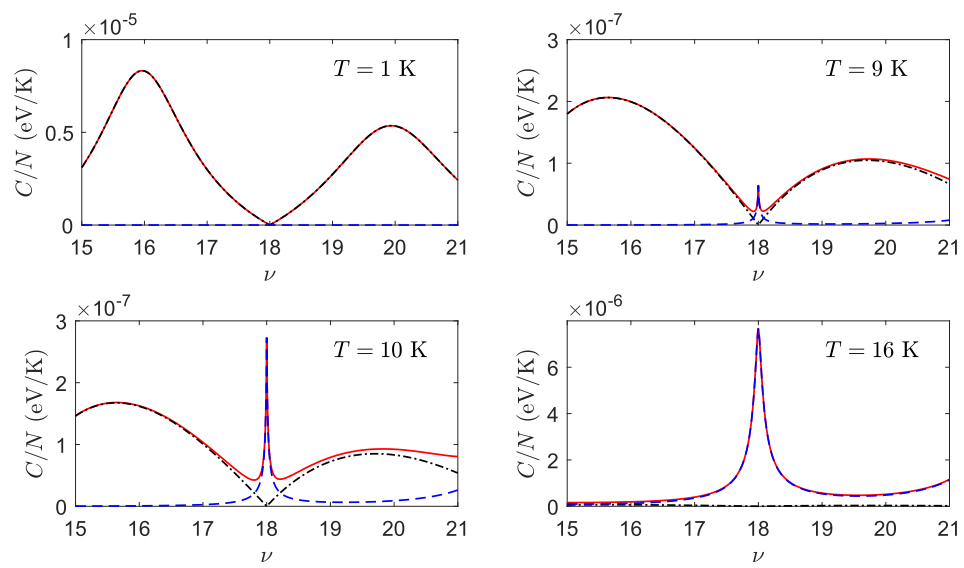

Figure 3. Electronic heat capacity as a function of the filling factor $\nu$, at different temperatures. The electron density is $n_{e}=10^{12} \mathrm{~cm}^{-2}$ with $N=10^{3}$ electrons. In black dot-dashed line we plot the heat capacity $C_{\mathrm{S}}$ due to the spin excitations, in blue dashed-line we plot the heat capacity $C_{\mathrm{L}}$ due to the different LLs excitations, and in red solid line we plot the total electronic heat capacity $C_{\mathrm{e}}=C_{\mathrm{S}}+C_{\mathrm{L}}$.

\section{Electronic heat capacity}

From the partition function, the electronic heat capacity (EHC) can be calculated as $C_{\mathrm{e}}=k_{\mathrm{B}} \beta^{2}\left(\partial^{2} \ln Z / \partial \beta^{2}\right)$. Given that $Z=e^{-\beta U} z_{\Theta} z_{\mathrm{S}} z_{\mathrm{L}}$, the EHC can be decomposed as $C_{\mathrm{e}}=C_{\mathrm{S}}+C_{\mathrm{L}}$, where

$$
C_{i}=k_{\mathrm{B}} \beta^{2}\left(\frac{\partial^{2} \ln z_{i}}{\partial \beta^{2}}\right) \quad i=\mathrm{S}, \mathrm{L}
$$

The term $C_{\mathrm{S}}$ gives the contribution due to the spin excitations in the last occupied LL, whereas $C_{\mathrm{L}}$ gives the contribution due to the excitations between the last LLs occupied in the ground state.

In Fig. 3 we plot the electronic heat capacity at different temperatures, as a function of the filling factor $\nu$, considering $n_{e}=10^{12} \mathrm{~cm}^{-2}$ and $N=10^{3}$ electrons. For all temperatures, in black dot-dashed line we plot $C_{\mathrm{S}}$, in blue dashed line we plot $C_{\mathrm{L}}$ and in red solid line we plot the total $\mathrm{EHC} C_{\mathrm{e}}=C_{\mathrm{S}}+C_{\mathrm{L}}$. As expected, the EHC oscillates as a function of the filling factor $\nu$. The maximum and minimum of the the oscillations are related to the type of thermal excitations. At very low temperatures the spin excitations dominate and the specific heat is entirely due to $C_{\mathrm{S}}$, as can be see at $T=1 \mathrm{~K}$. The EHC is maximum at values $\nu=16$ and $\nu=20$, which corresponds to the half filling of the last occupied LL (see Fig. 1). In that case, one spin is full and the other is empty, so the possible spin excitations are maximum. As the temperature increases, a new peak appears in $C_{\mathrm{e}}$ around $\nu=18$, caused by the contribution of $C_{\mathrm{L}}$. The EHC becomes maximum at values $\nu=2(2 n+1)$, which correspond to full filling of the LLs. This is expected, as more states are available when the electrons are thermally excited from a full LL to an empty LL. At $T=9 \mathrm{~K}$ and $T=10 \mathrm{~K}$ we see the transition in which both the spin and LL contribute to the EHC. However, at higher temperatures $C_{\mathrm{L}}$ always dominates and the EHC practically reduces to $C_{\mathrm{L}}$, as can be seen at $T=16 \mathrm{~K}$. In this 


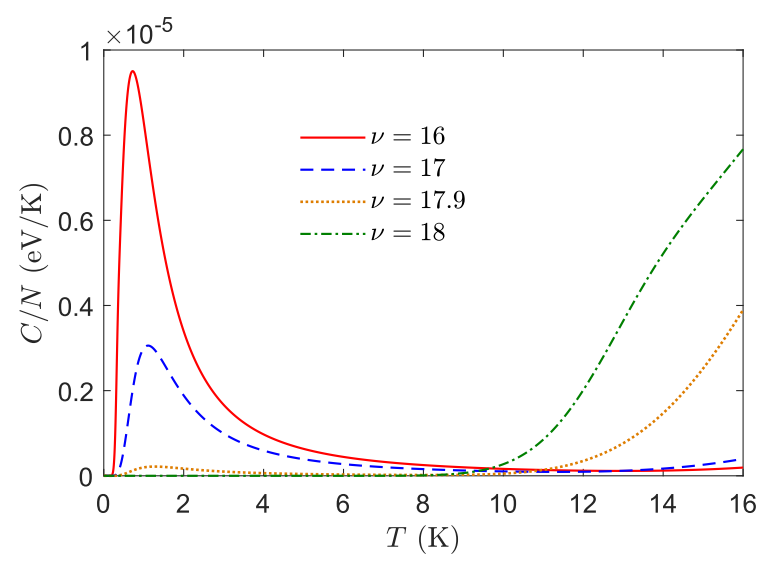

Figure 4. Electronic heat capacity as a function of the temperature, for different filling factors $\nu$. The electron density is $n_{e}=10^{12} \mathrm{~cm}^{-2}$ with $N=10^{3}$ electrons.

stage, the specific heat at all $\nu$ is due only to excitations between different LLs, and thus the spin splitting can be neglected.

The transition between the EHC due to intra-LLs or inter-LLs excitations, as shown in Fig. 3, can be useful to determine the energy levels dependence on the spin splitting. Indeed, the profile of the oscillations depends on the Zeeman energy, which in turn depends on the g-factor in graphene. Moreover, the EHC due to inter-LLs excitations depends on the cyclotron energy. Hence careful measurements of the heat capacity oscillations under a magnetic field can elucidate the properties of the relativistic Landau levels in graphene.

The specific heat dependence on the spin and LL excitations can also be seen in Fig. 4, where we plot $C_{\mathrm{e}}$ as a function the temperature, at different filling factors $\nu$, considering $n_{e}=10^{12} \mathrm{~cm}^{-2}$ and $N=10^{3}$ electrons. At low temperatures the EHC is entirely due to $C_{\mathrm{S}}$ and we clearly see the Schottky anomaly effect, with the EHC rising to a maximum and then decreasing. Such behaviour is consistent with the EHC being due to discrete energy levels, which in this case are just the excitation energies $\Delta E=2 \mu_{\mathrm{B}} B$. Notice that the magnitude of the Schottky anomaly decreases as the filling factor changes, being maximum at $\nu=16$ and zero at $\nu=18$ (no possible spin excitations). At higher temperatures we observe an increase in $C_{\mathrm{e}}$ due to the contribution of $C_{\mathrm{L}}$, caused by the inter-LLs excitations. The dependence of $C_{\mathrm{e}}$ with $\nu$ is now reversed with respect to the low temperature case: the EHC is maximum at $\nu=18$, when the thermal excitations between different LLs is higher.

\subsection{Comparison with the $E H C$ at $B=0$}

It is instructive to compare the the EHC under magnetic fields with the EHC at $B=0$ in doped graphene. Without magnetic fields, the conduction band density of states per area is $\rho(\varepsilon)=2 \varepsilon / \pi \hbar^{2} v_{\mathrm{F}}^{2}$, taking into account the valley and spin degeneracy [9]. In the 
Sommerfeld approximation [36] the energy is

$$
U \simeq \int_{0}^{\mu} \rho(\varepsilon) \varepsilon d \varepsilon+\left.\frac{\pi^{2}}{6}\left(k_{\mathrm{B}} T\right)^{2} \frac{d(\rho \varepsilon)}{d \varepsilon}\right|_{\varepsilon=\mu},
$$

where the chemical potential is obtained from the condition

$$
n_{e}=\int_{0}^{\mu} \rho(\varepsilon) d \varepsilon+\left.\frac{\pi^{2}}{6}\left(k_{\mathrm{B}} T\right)^{2} \frac{d \rho}{d \varepsilon}\right|_{\varepsilon=\mu} .
$$

Solving for $\mu$ we get $\mu^{2}=\varepsilon_{\mathrm{F}}^{2}-\pi^{2}\left(k_{\mathrm{B}} T\right)^{2} / 3$, where $\varepsilon_{\mathrm{F}}=\hbar v_{\mathrm{F}} \sqrt{\pi n_{e}}$ is the Fermi energy determined by $n_{e}=\int_{0}^{\varepsilon_{F}} \rho(\varepsilon) d \varepsilon$. At low temperatures and usual densities $n_{e} \sim 10^{12} \mathrm{~cm}^{-2}$ we have $\varepsilon_{\mathrm{F}} \gg k_{\mathrm{B}} T$, so the chemical potential hardly changes with the temperature. Then we can take $\mu=\varepsilon_{\mathrm{F}}$ and the EHC is

$$
\frac{C_{\mathrm{e}}}{N}(B=0)=\frac{4}{3} \pi^{2} k_{\mathrm{B}}^{2} \frac{T}{\varepsilon_{\mathrm{F}}} .
$$

For $n_{e}=10^{12} \mathrm{~cm}^{-2}$ we have $\varepsilon_{\mathrm{F}} \simeq 0.012 \mathrm{eV}$ and $C_{\mathrm{e}}(B=0) / N \simeq 8 \times 10^{-7} T[\mathrm{~K}] \mathrm{eV} / \mathrm{K}$, which at low temperatures is much smaller than the EHC oscillation peaks at strong magnetic fields. For instance, at $T=1 \mathrm{~K}$, for $\nu=16$ the spin induced EHC is about $10^{-5} \mathrm{eV} / \mathrm{K}$ (see Fig. 4), so $C_{\mathrm{e}}(\nu=16) / C_{\mathrm{e}}(B=0) \sim 10$. However, this ratio decreases at higher temperatures, even at values $\nu=2(2 n+1)$ where the inter-LLs excitations are maximum.

\subsection{Comparison with the phonon heat capacity}

In graphene the phonon contribution to the heat capacity dominates, even at very low temperatures [14. Thus it is important to take in consideration this contribution in order to have an estimation of the oscillation amplitudes. Under the harmonic approximation, the low temperature phonon heat capacity is dominated by the acoustic modes. These are the two in-plane vibration LA and TA modes (with $\omega \propto k$ and $C_{\mathrm{p}} \propto T^{2}$ ), and the transverse ZA mode (with $\omega \propto k$ and $C_{\mathrm{p}} \propto T$ ). However, at low temperatures the ZA mode always dominates. The ZA mode heat capacity is [16]

$$
C_{\mathrm{p}}=\frac{\pi}{12} \frac{k_{\mathrm{B}}^{2}}{\hbar} A_{0} \sqrt{\frac{\rho}{\kappa}} T
$$

where $A_{0} \simeq 2.62 \AA^{2} /$ atom is the graphene surface area, $\rho$ is the surface density and $\kappa$ is the bending constant for the ZA phonon band $(\kappa=1.49 \mathrm{eV})$. This gives $C_{\mathrm{p}} / N=a T$, where $a \simeq 5.27 \times 10^{-4} \mathrm{eV} / \mathrm{K}^{2}$ for $n_{e}=10^{12} \mathrm{~cm}^{-2}$. Thus, even at $T=1 \mathrm{~K}$, the phonon heat capacity is orders of magnitude bigger than the electronic heat capacity.

In Fig. 5 we show the heat capacity ratio $C_{\mathrm{e}} / C_{\mathrm{p}}$ in the filling factor/temperature plane, for $n_{e}=10^{12} \mathrm{~cm}^{-2}$ with $N=10^{3}$ electrons. Given that $\nu=n_{e} \phi / B$, the filling factors correspond to magnetic fields from $B \simeq 4.1 \mathrm{~T}(\nu=10)$ to $B \simeq 1.9 \mathrm{~T}(\nu=22)$. At the low temperatures considered we have $C_{\mathrm{e}}=C_{\mathrm{S}}$ so the ratio $C_{\mathrm{e}} / C_{\mathrm{p}}$ is maximum at values $\nu=12,16,20$, i.e. half filling of the last $\mathrm{LL}$ occupied. The maximum oscillating amplitude is approximately $3 \%$ of $C$, which is about the same order of the specific 


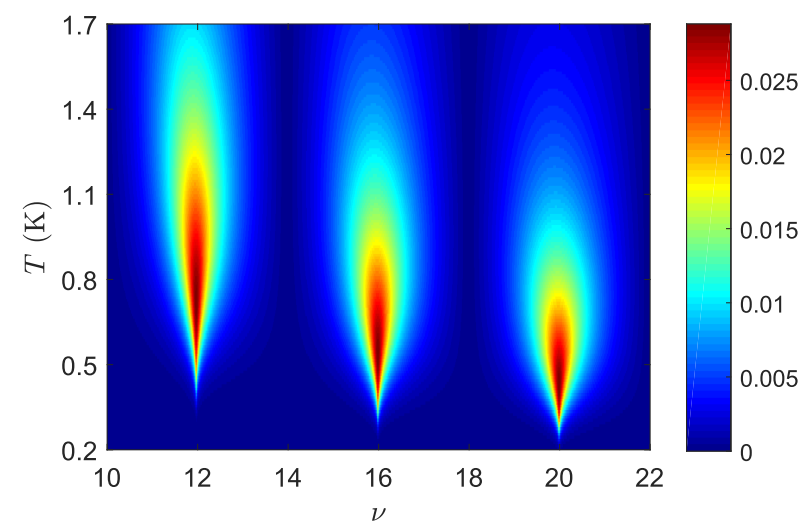

Figure 5. Heat capacity ratio $C_{\mathrm{e}} / C_{\mathrm{p}}$ between the electronic and phonon contributions, in the filling factor/temperature plane. The electron density is $n_{e}=10^{12} \mathrm{~cm}^{-2}$ with $N=10^{3}$ electrons.

heat oscillations measured in Be [37]. However, the range of decay in $C_{\mathrm{e}} / C_{\mathrm{p}}$ is larger in temperature at lower filling factors. In other words, the Schottky anomaly is more spread at low $\nu$.

\subsection{Maximum amplitude of oscillation}

Fig. 5 seems to suggest that the heat capacity peaks have a maximum amplitude independent of $\nu$. We shall now show that this is indeed the case. Since we will be considering fixed integer values of $\nu$, the analysis is greatly simplified by working in the grand canonical ensemble. We will treat separately the maxima in $C_{\mathrm{S}}$ and $C_{\mathrm{L}}$.

At the low temperatures in which the spin excitations dominate, only the occupation of the last LL (for each spin) changes with the temperature. If, in general, the last LL occupied is $\varepsilon_{0}$, the average occupation for each spin is

$$
n_{ \pm}=\frac{D}{1+e^{\beta\left(\varepsilon_{0} \pm \mu_{B} B-\mu\right)}} .
$$

The chemical potential must obey the fixed electron density condition. Considering that the LLs $\varepsilon_{n}<\varepsilon_{0}$ are fully occupied and the LLs $\varepsilon_{n}>\varepsilon_{0}$ empty, we have

$$
\frac{n_{e}}{n_{B}}=\nu-1+\sum_{s= \pm 1} \frac{1}{1+e^{\beta\left(\varepsilon_{0}+s \mu_{\mathrm{B}} B-\mu\right)}} .
$$

Hence $\mu=\varepsilon_{0}$, which means that at half filling the chemical potential lies exactly between the spin splitting of the LL. The average energy is

$$
U=U(0)+D \sum_{s= \pm 1} \frac{\varepsilon_{0}+s \mu_{\mathrm{B}} B}{1+e^{\beta s \mu_{\mathrm{B}} B}}
$$

where $U(0)$ is the temperature independent contribution of the lower filled LL. Therefore the spin heat capacity at half filling reads

$$
\frac{C_{\mathrm{S}}^{\mathrm{hf}}}{N}=k_{\mathrm{B}}\left(\frac{\beta \mu_{\mathrm{B}} n_{e} \phi}{\nu^{3 / 2}}\right)^{2} \operatorname{sech}^{2}\left(\frac{\beta \mu_{\mathrm{B}} n_{e} \phi}{2 \nu}\right),
$$


where we replaced $D / N=2 / \nu$ and $B=n_{B} \phi=n_{e} \phi / \nu(\phi=h / e$ is the magnetic flux). This expression for $C_{\mathrm{S}}^{\mathrm{hf}}$ clearly shows the Schottky anomaly effect as the temperature changes. The maximum of $C_{\mathrm{S}}^{\mathrm{hf}}$ is determined by $\partial C_{\mathrm{S}}^{\mathrm{hf}} / \partial \beta=0$, which implies $x \tanh x=1$, where $x=\beta \mu_{\mathrm{B}} n_{e} \phi / 2 \nu$. This has a numerical solution $x \simeq 6 / 5$, so the maxima in $C_{\mathrm{S}}^{\mathrm{hf}}$ occur at temperatures

$$
T_{m}=\frac{5}{12} \frac{\mu_{\mathrm{B}} n_{e} \phi}{\nu k_{\mathrm{B}}}
$$

which depend linearly with the magnetic field, as in undoped graphene [27]. Replacing in equation (18) we obtain

$$
\frac{C_{\mathrm{S}}^{\mathrm{hf}}}{N} \stackrel{\max }{=} \frac{44}{25} \frac{k_{\mathrm{B}}}{\nu} .
$$

For $\nu=16$ we get $C_{\mathrm{S}}^{\mathrm{hf}} / N \stackrel{\max }{\simeq} 10^{-5} \mathrm{eV} / \mathrm{K}$, in agreement with Fig. 4 .

The maximum of the ratio between the electronic and phonon heat capacity can be found in a similar way. The condition $\partial\left(C_{\mathrm{S}}^{\mathrm{hf}} / C_{\mathrm{p}}\right) / \partial \beta=0$ implies $x \tanh x=3 / 2$ $\left(x=\beta \mu_{\mathrm{B}} n_{e} \phi / 2 \nu\right)$, which has a numerical solution $x \simeq 1.622$. Thus there is a slight shift of the maximum temperature, in comparison to the maximum in $C_{\mathrm{S}}^{\mathrm{hf}}$. Specifically, the maxima in $C_{\mathrm{S}}^{\mathrm{hf}} / C_{\mathrm{p}}$ occur at temperatures $T_{M}=(6 / 5) T_{m} / 1.622 \simeq 0.74 T_{m}$. The maximum ratio reads

$$
\frac{C_{\mathrm{S}}^{\mathrm{hf}}}{C_{\mathrm{p}}} \stackrel{\max }{\simeq} \frac{30}{\pi^{2}} \frac{e}{\mu_{\mathrm{B}}} \sqrt{\frac{\kappa}{\rho}} .
$$

For $\kappa=1.49 \mathrm{eV}$ [16] we get $C_{\mathrm{S}}^{\mathrm{hf}} / C_{\mathrm{p}} \stackrel{\max }{\simeq} 0.03$, in agreement with Fig. 5. Notice that the maximum ratio of $C_{\mathrm{S}}^{\mathrm{hf}} / C_{\mathrm{p}}$ does not depend on the magnetic field, the electron density or the temperature, it depends only on the intrinsic properties of the lattice and the electrons.

The same analysis can be made for the maximum amplitude in the EHC due to inter-LLs excitations, which occur at full filling $\nu=2 n(2+n)$. Then we only consider the thermal excitations $\varepsilon_{q_{0}} \rightarrow \varepsilon_{q_{0}+1}$, where $\varepsilon_{q_{0}}$ is the last LL fully occupied in the ground state and $q_{0}=(\nu-2) / 4$. Neglecting the spin splitting as we did in Sec. 2.2. the occupation is $n_{i}=2 D\left[1+e^{\beta\left(\varepsilon_{i}-\mu\right)}\right]^{-1}$, where we defined $\varepsilon_{1}=\varepsilon_{q_{0}}$ and $\varepsilon_{2}=\varepsilon_{q_{0}+1}$. As in the spin excitations case, the chemical potential is determined by $n_{e} / n_{B}=\nu-1+\left(n_{1}+n_{2}\right) / 2 D$, which implies $\mu=\left(\varepsilon_{2}+\varepsilon_{1}\right) / 2$. Thus $\mu$ lies between the energy levels $\varepsilon_{q_{0}}$ and $\varepsilon_{q_{0}}+1$. Consequently a similar expression is found for the heat capacity at full filling

$$
\frac{C_{\mathrm{L}}^{\mathrm{ff}}}{N}=\frac{k_{\mathrm{B}}}{2 \nu}(\beta \Delta \varepsilon)^{2} \operatorname{sech}^{2}\left(\frac{\beta \Delta \varepsilon}{4}\right),
$$

where $\Delta \varepsilon \equiv \varepsilon_{q_{0}+1}-\varepsilon_{q_{0}}$. The expression of $C_{\mathrm{L}}^{\mathrm{ff}}$ is similar to equation (18) but with the LL difference $\Delta \varepsilon$ instead of the Zeeman energy $2 \mu_{\mathrm{B}} B$. Therefore the same analysis can be made to obtain the maxima in $C_{\mathrm{L}}^{\mathrm{ff}}$. However, we are only interested in the maximum of the ratio $C_{\mathrm{L}}^{\mathrm{ff}} / C_{\mathrm{p}}$. Following the same procedure to obtain equation (21), we get

$$
\frac{C_{\mathrm{L}}^{\mathrm{ff}}}{C_{\mathrm{p}}} \stackrel{\max }{\simeq} \frac{120}{\nu \pi^{2}} \frac{h n_{e}}{\Delta \varepsilon} \sqrt{\frac{\kappa}{\rho}} .
$$


In contrast to the spin excitation case, the maximum of $C_{\mathrm{L}}^{\mathrm{ff}} / C_{\mathrm{p}}$ depends on the electron density and the filling factor. However, when $\nu \gg 1$ we have $\Delta \varepsilon \simeq 2 \hbar v_{\mathrm{F}} \sqrt{n_{e}} / \nu$ and the ratio depends only on the electron density

$$
\frac{C_{\mathrm{L}}^{\mathrm{ff}}}{C_{\mathrm{p}}} \stackrel{\max }{\simeq} \frac{120}{\pi^{2} v_{\mathrm{F}}} \sqrt{\pi n_{e} \frac{\kappa}{\rho}} \quad(\nu \gg 1) .
$$

For $n_{e}=10^{12} \mathrm{~cm}^{-2}$ we get $C_{\mathrm{L}}^{\mathrm{ff}} / C_{\mathrm{p}} \stackrel{\max }{\simeq} 1.2 \times 10^{-3}$, which is one order of magnitude lower that the maximum of the ratio $C_{\mathrm{S}}^{\mathrm{hf}} / C_{\mathrm{p}}$. Hence at high temperatures, when the inter-LLs excitations dominate, the EHC is at maximum three order of magnitude lower than the phonon heat capacity [28]. This is in fact similar to the behaviour without magnetic fields [14], where $C_{\mathrm{e}} / C_{\mathrm{p}} \sim 10^{-3}$ [see Eqs. (13) and (14)].

\subsection{Impurities effect}

So far we have assume pristine graphene, i.e. without impurities. However, in the practice systems often contain impurities so it may be important to study their effect in the EHC, particularly in the maximum amplitude of oscillation. Under a magnetic field, the effect of the impurities is to broaden each LL density of states (DOS). The type of broadening distribution, and the magnitude of the broadening, depends on the scattering mechanism of electrons under a magnetic field. In graphene the broadening can depend significantly on the LL itself [38], especially at low occupancy. Thus, given the problem complexity, the following discussion will be more qualitatively.

For simplicity we consider the impurities effect only on the spin-induced heat capacity (i.e. intra-LL thermal excitations), which have the higher amplitude of oscillation in relation to $C_{\mathrm{p}}$. Following the discussion in section 3.3, we will calculate the EHC at half filling by working in the grand canonical ensemble. We shall assume a broadening parameter $\Gamma$ such that $\Gamma / \Delta \varepsilon_{\mathrm{L}} \ll 1$, so at low temperatures the intra-LL excitations still dominate. Then if the last occupied LL is $\varepsilon_{0}$, with impurities the average occupation for each spin is

$$
n_{ \pm}=D \int_{-\infty}^{\infty} \rho\left(\varepsilon-\varepsilon_{0} \mp \mu_{\mathrm{B}} B\right) \frac{1}{1+e^{\beta(\varepsilon-\mu)}} d \varepsilon,
$$

where $\rho\left(\varepsilon-\varepsilon_{0} \mp \mu_{\mathrm{B}} B\right)$ is the broadening distribution. Assuming $\rho$ to be symmetric, at half filling the constant electron density condition still implies $\mu=\varepsilon_{0}$, as in the pristine case. Then the average energy reads

$$
U=U(0)+2 D \int_{-\infty}^{\infty} \frac{\rho\left(x-\mu_{\mathrm{B}} B\right)}{1+e^{\beta x}} x d x,
$$

where again $U(0)$ is the temperature independent contribution of the lower occupied LLs, while in the last integral we changed variables $x=\varepsilon-\varepsilon_{0}$ and used the fact that $\rho\left(x \pm \mu_{B} B\right)$ are symmetric around $x=0$. Hence the heat capacity at half filling becomes

$$
\frac{C_{\mathrm{S}}^{\mathrm{hf}}}{N}=\frac{k_{\mathrm{B}} \beta^{2}}{\nu} \int_{-\infty}^{\infty} \rho\left(x-\frac{\mu_{\mathrm{B}} n_{e} \phi}{\nu}\right) x^{2} \operatorname{sech}^{2}\left(\frac{\beta x}{2}\right) d x
$$



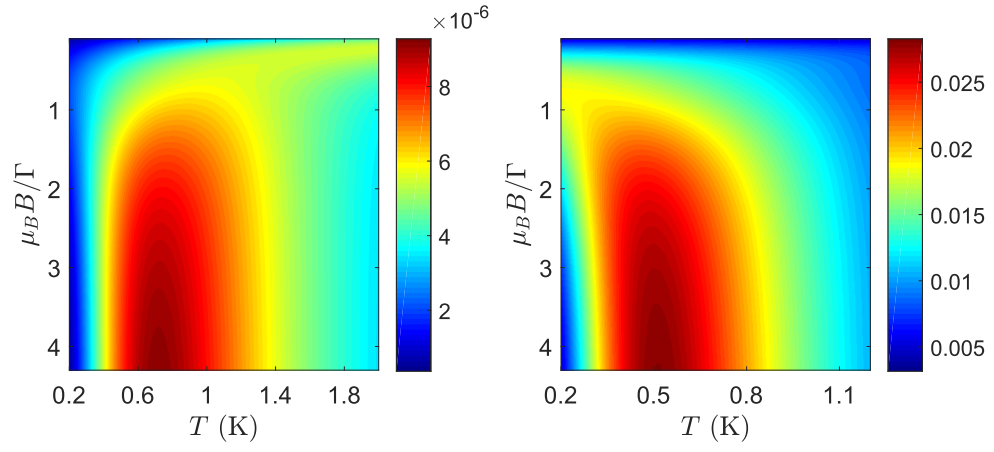

Figure 6. Heat capacity (a) $C_{\mathrm{S}} / N$ (in $\mathrm{eV} / \mathrm{K}$ ) and (b) ratio $C_{\mathrm{S}} / C_{\mathrm{p}}$ between the electronic and phonon contributions, in the temperature/broadening plane, for the filling factor $\nu=16$. The electron density is $n_{e}=10^{12} \mathrm{~cm}^{-2}$. The impurities have a Gauss distribution with a broadening parameter $\Gamma$.

where we replaced $D / N=2 / \nu$ and $B=n_{B} \phi=n_{e} \phi / \nu$. Notice that the last equation reduces to equation (18) if $\rho \rightarrow \delta$, where $\delta$ is the Dirac delta. Therefore impurities tend to reduce and spread the Schottky anomaly. This is of course the expected result, as impurities act much like the temperature effect by damping the oscillations.

In Fig. 6 we show (a) $C_{\mathrm{S}}^{\mathrm{hf}} / N($ in $\mathrm{eV} / \mathrm{K})$ and the ratio (b) $C_{\mathrm{S}}^{\mathrm{hf}} / C_{\mathrm{S}}$, for $\nu=16$, in the temperature/broadening plane, with $n_{e}=10^{12} \mathrm{~cm}^{-2}$ and $N=10^{3}$. We consider impurities with a Gauss distribution $\rho=\exp \left[-\left(x-\mu_{\mathrm{B}} n_{e} \phi / \nu\right)^{2} / \Gamma^{2}\right] / \Gamma \sqrt{\pi}$, although in principle any other symmetric distribution could be used. As we see in Fig. 6(a), the EHC is reduced as the broadening $\Gamma$ increases. For a Gauss distribution, the width of the DOS $\rho$ is approximately $2 \Gamma$. Then we can see a different behaviour depending on the relation of $\Gamma$ with the splitting energy $\mu_{\mathrm{B}} B$. First, when $2 \Gamma<\mu_{\mathrm{B}} B$ the DOS for each spin do not overlap and the impurity effect is relatively small. Therefore $C_{\mathrm{S}}$ still remains dominated by the spin excitations. In particular, in Fig 6(b) we observe that the maximum amplitude of $C_{\mathrm{S}}^{\mathrm{hf}} / C_{\mathrm{S}}$ still is approximately $3 \%$ of $C_{\mathrm{p}}$, as in the pristine case. When $\mu_{\mathrm{B}} B<2 \Gamma<2 \mu_{\mathrm{B}} B$ the DOS start to mix and the EHC maximum amplitude decreases more pronouncedly as the broadening increases. We also begin to see a shift to lower temperatures for the maximum ratio of $C_{\mathrm{S}}^{\mathrm{hf}} / C_{\mathrm{S}}$. Finally, when $\Gamma>\mu_{\mathrm{B}} B$ the DOS width for each spin is bigger than the Zeeman energy and hence the spin splitting is practically damped by the impurities. Consequently the EHC is reduced quite abruptly. In this situation the spin splitting could in fact be directly ignored, with the intra-LL excitations being accounted only by the impurities effect [39].

Similar behaviour is expected for the impurity effect on the EHC due to inter-LLs excitations, if the broadening is comparable to the LLs energy difference. In particular, as the broadening increases, the inter-LLs thermal excitations become appreciable at lower temperatures. However, the maximum amplitude in the EHC is lowered with increasing impurities, so the heat capacity oscillations could become negligible in comparison to the phonon contribution. 


\section{Conclusions}

We studied the low temperature electronic heat capacity in doped graphene under perpendicular magnetic fields. The partition function and the heat capacity were obtained analytically considering the excitations between the last occupied energy levels in the ground state. At low temperatures, the specific heat is dominated by the spin excitations in the last occupied LL. Then the heat capacity oscillates with the magnetic field, being maximum at half filling, when more spins can be excited. At constant temperature the Schottky anomaly is observed, with a magnitude strongly dependent on the filling factor. At higher temperatures, the heat capacity increases due to interLLs excitations. The increase is maximum at full filling, when the thermal excitations are from the last LL fully occupied to the next empty LL.

The magnitude of the heat capacity oscillations were compared with the phonon contribution $C_{\mathrm{p}}$. At low temperatures the spin-induced heat capacity has a maximum amplitude approaching $3 \%$ of $C_{\mathrm{p}}$, independently of the filling factor and the temperature. In contrast, the maximum oscillation amplitude due to inter-LLs excitations is much smaller, about $0.1 \%$ of $C_{\mathrm{p}}$ for usual electron densities. These oscillation amplitudes decrease in the presence of impurities, although the effect depends on the magnitude of the LLs broadening $\Gamma$. In particular, if $2 \Gamma<\mu_{\mathrm{B}} B$ then the spin excitations still dominate the EHC at low temperatures, whereas if $\Gamma>\mu_{\mathrm{B}} B$ the $\mathrm{EHC}$ is dominated by intra-LLs excitations induced by impurities, with a lower amplitude than in the pristine case.

\section{Acknowledgments}

This paper was partially supported by grants of CONICET (Argentina National Research Council) and Universidad Nacional del Sur (UNS) and by ANPCyT through PICT 2014-1351. Res. N 270/15. N: 2014-1351, and PIP 2014-2016. Res. N 5013/14. Código: 11220130100436CO research grant, as well as by SGCyT-UNS., J. S. A. and P. J. are members of CONICET, F. E. acknowledge research fellowship from this institution.

\section{Appendix A. High temperature limit of the spin excitations}

At high temperatures such that $e^{-2 \beta \mu_{\mathrm{B}} B} \simeq 1$, equation (5) reduces to $z_{\mathrm{S}} \simeq$ ${ }_{2} F_{1}\left(-D,-D_{q+1}, D_{q}+1,1\right)$. Using the property

$$
{ }_{2} F_{1}(a, b, c, 1)=\frac{\Gamma(c) \Gamma(c-a-b)}{\Gamma(c-a) \Gamma(c-b)},
$$

we obtain

$$
z_{\mathrm{S}} \simeq \frac{\Gamma\left(D_{q}+1\right) \Gamma(2 D+1)}{\Gamma\left(D+D_{q}+1\right) \Gamma(D+1)},
$$


where we used $D_{q}+D_{q+1}=D$. Then, given that $z_{\Theta}=\left(\begin{array}{c}D \\ D \Theta\end{array}\right)$ can be generally written as $z_{\Theta}=\Gamma(D+1) / \Gamma\left(D_{q}+1\right) \Gamma\left(D_{q+1}+1\right)$, we obtain

$$
z_{\Theta} z_{\mathrm{S}} \simeq \frac{\Gamma(2 D+1)}{\Gamma\left(D+D_{q}+1\right) \Gamma\left(D-D_{q}+1\right)} .
$$

Now, if $q$ is odd then the last partially occupied LL has spin up, whereas if $q$ is even the last partially occupied LL has spin down. The partial occupancy, in the ground state, is determined by $\Theta=\nu / 2$ - floor $[\nu / 2]$. Without spin splitting, each LL has degeneracy $2 D$ and the partial occupancy is given by $\Theta_{0}=\nu_{G} / 4-$ floor $\left[\nu_{G} / 4\right]$, where $\nu_{G}=\nu-2$. When $q$ is odd we have $\Theta_{0}=\Theta / 2$ whereas if $q$ is even we have $\Theta_{0}=\Theta / 2+1 / 2$. Therefore $D_{q}=D-2 D \Theta_{0}$ if $q$ is odd and $D_{q}=2 D \Theta_{0}-D$ if $q$ is even. Thus $z_{\Theta} z_{\mathrm{S}} \simeq z_{\Theta_{0}}$, where $z_{\Theta_{0}}=\left(\begin{array}{c}2 D \\ 2 D \Theta_{0}\end{array}\right)$. This proves that, at high temperature, the spin excitations between the same LL reduce to the number of ways the last LL can be partially occupied, if one ignores the spin splitting.

\section{References}

[1] Geim A K and Novoselov K S 2007 Nature Materials 6 183-191

[2] Gupta A, Sakthivel T and Seal S 2015 Progress in Materials Science $\mathbf{7 3} 44-126$

[3] Lee C, Wei X, Kysar J W and Hone J 2008 Science 321 385-388

[4] Sakhaee-Pour A 2009 Solid State Communications 149 91-95

[5] Kalosakas G, Lathiotakis N N, Galiotis C and Papagelis K 2013 Journal of Applied Physics 113 134307

[6] Chen H, Mller M B, Gilmore K J, Wallace G G and Li D 2008 Advanced Materials 20 3557-3561

[7] Horng J, Chen C F, Geng B, Girit C, Zhang Y, Hao Z, Bechtel H A, Martin M, Zettl A, Crommie M F, Shen Y R and Wang F 2011 Physical Review B 83165113

[8] Novoselov K S, Geim A K, Morozov S V, Jiang D, Katsnelson M I, Grigorieva I V, Dubonos S V and Firsov A A 2005 Nature 438 197-200

[9] Neto A H C, Guinea F, Peres N M R, Novoselov K S and Geim A K 2009 Reviews of Modern Physics 81 109-162

[10] Reich S, Maultzsch J, Thomsen C and Ordejón P 2002 Physical Review B 66035412

[11] Sprinkle M, Siegel D, Hu Y, Hicks J, Tejeda A, Taleb-Ibrahimi A, Fèvre P L, Bertran F, Vizzini S, Enriquez H, Chiang S, Soukiassian P, Berger C, de Heer W A, Lanzara A and Conrad E H 2009 Physical Review Letters 103226803

[12] Hwang C, Siegel D A, Mo S K, Regan W, Ismach A, Zhang Y, Zettl A and Lanzara A 2012 Scientific Reports $\mathbf{2}$

[13] Balandin A A 2011 Nature Materials 10 569-581

[14] Pop E, Varshney V and Roy A K 2012 MRS Bulletin 37 1273-1281

[15] Renteria J, Nika D and Balandin A 2014 Applied Sciences 4 525-547

[16] Herrero C P and Ramírez R 2018 The Journal of Chemical Physics 148102302

[17] Benedict L X, Louie S G and Cohen M L 1996 Solid State Communications 100 177-180

[18] Goerbig M O 2011 Reviews of Modern Physics 83 1193-1243

[19] Bhandari S, Lee G H, Klales A, Watanabe K, Taniguchi T, Heller E, Kim P and Westervelt R M 2016 Nano Letters 16 1690-1694

[20] Novoselov K S, Jiang Z, Zhang Y, Morozov S V, Stormer H L, Zeitler U, Maan J C, Boebinger G S, Kim P and Geim A K 2007 Science 315 1379-1379

[21] Jiang Z, Zhang Y, Tan Y W, Stormer H and Kim P 2007 Solid State Communications 143 14-19

[22] Safeer C K, Ingla-Aynés J, Herling F, Garcia J H, Vila M, Ontoso N, Calvo M R, Roche S, Hueso L E and Casanova F 2019 Nano Letters 19 1074-1082 
[23] Shoenberg D 1984 Magnetic Oscillations in Metals (Cambridge University Press)

[24] Escudero F, Ardenghi J S and Jasen P 2020 The European Physical Journal B 93

[25] Ando T 1974 Journal of the Physical Society of Japan 37 1233-1237

[26] Escudero F, Ardenghi J and Jasen P 2018 Journal of Magnetism and Magnetic Materials 454 $131-138$

[27] Lin S Y, Ho Y H, Huang Y C and Lin M F 2012 Journal of the Physical Society of Japan 81 084602

[28] Alisultanov Z and Reis M 2016 Physics Letters A 380 470-474

[29] Shoenberg D 1984 Journal of Low Temperature Physics 56 417-440

[30] Champel T 2001 Physical Review B 64054407

[31] Nasir R, Khan M A, Tahir M and Sabeeh K 2009 Journal of Physics: Condensed Matter 22025503

[32] Escudero F, Ardenghi J, Sourrouille L and Jasen P 2017 Journal of Magnetism and Magnetic Materials 429 294-298

[33] Higuchi M, Hamal D B, Shrestha A and Higuchi K 2019 Journal of the Physical Society of Japan 88094707

[34] Grazianetti C, Cinquanta E and Molle A 2016 2D Materials 3012001

[35] Zhang Y, Rubio A and Lay G L 2017 Journal of Physics D: Applied Physics 50053004

[36] Sommerfeld A 1928 Zeitschrift fr Physik 47 1-32

[37] Sullivan P F and Seidel G 1968 Physical Review 173 679-685

[38] Yang C H, Peeters F M and Xu W 2010 Physical Review B 82075401

[39] Zawadzki W and Lassnig R 1984 Surface Science 142 225-235 